\title{
Ovarian cycling and reproductive state shape the vaginal microbiota in wild baboons
}

\author{
Elizabeth A. Miller ${ }^{1 *}$, Joshua A. Livermore ${ }^{1}$, Susan C. Alberts ${ }^{2,4}$, Jenny Tung ${ }^{2,3,45}$ and Elizabeth A. Archie $e^{1,2}$
}

\begin{abstract}
Background: The vaginal microbiome is an important site of bacterial-mammalian symbiosis. This symbiosis is currently best characterized for humans, where lactobacilli dominate the microbial community and may help defend women against infectious disease. However, lactobacilli do not dominate the vaginal microbiota of any other mammal studied to date, raising key questions about the forces that shape the vaginal microbiome in nonhuman mammals.

Results: We used Illumina sequencing of the bacterial 165 rRNA gene to investigate variation in the taxonomic composition of the vaginal microbiota in 48 baboons (Papio cynocephalus), members of a well-studied wild population in Kenya. Similar to prior studies, we found that the baboon vaginal microbiota was not dominated by lactobacilli. Despite this difference, and similar to humans, reproductive state was the dominant predictor of baboon vaginal microbiota, with pregnancy, postpartum amenorrhea, and ovarian cycling explaining $18 \%$ of the variance in community composition. Furthermore, among cycling females, a striking $39 \%$ of variance in community composition was explained by ovarian cycle phase, with an especially distinctive microbial community around ovulation. Periovulatory females exhibited the highest relative abundance of lactic acid-producing bacteria compared to any other phase, with a mean relative abundance of $44 \%$. To a lesser extent, sexual behavior, especially a history of shared sexual partners, also predicted vaginal microbial similarity between baboons.

Conclusions: Despite striking differences in their dominant microbes, both human and baboon vaginal microbiota exhibit profound changes in composition in response to reproductive state, ovarian cycle phase, and sexual behavior. We found major shifts in composition during ovulation, which may have implications for disease risk and conception success. These findings highlight the need for future studies to account for fine-scale differences in reproductive state, particularly differences between the various phases of the ovarian cycle. Overall, our work contributes to an emerging understanding of the forces that explain intra- and inter-individual variation in the mammalian vaginal microbiome, with particular emphasis on its role in host health and disease risk.
\end{abstract}

Keywords: Vaginal microbiome, Primate, Reproductive state, Ovulation, Transmission

\section{Background}

The composition of the human vaginal microbiome varies considerably between individuals and within the same individual over time $[1,2]$. Such variation is important because it can have major consequences for a woman's vaginal health, disease risk, and fertility [3-5]. In contrast to humans, we know very little about the

\footnotetext{
*Correspondence: emille18@nd.edu

'Department of Biological Sciences, University of Notre Dame, Notre Dame, IN, USA

Full list of author information is available at the end of the article
}

causes and consequences of inter-individual variation in the vaginal microbiomes of non-human mammals. Furthermore, human vaginal microbiomes differ considerably from those of other mammals, including other primates [6], raising key questions about whether the forces that shape the human vaginal microbiome are unique to humans or are shared with other primates or mammals. Answering this question is important to understanding both (i) the generalizability of factors that explain inter-individual variation in the vaginal microbiome in different species and (ii) the ways in which 
non-human primates can serve as useful models for human vaginal microbial communities.

Unlike other primates, the human vaginal microbiota is usually dominated by members of the genus Lactobacillus, which typically comprise $70 \%$ or more of resident bacteria $[1,2]$. Lactobacilli dominance is important for understanding both the forces shaping the human vaginal microbiome as well as its hypothesized functional properties. Specifically, lactobacilli produce lactic acid from the breakdown products of glycogen (e.g., maltose) in vaginal fluid [7-13]. This reaction creates an acidic environment $(\mathrm{pH} \leq 4.5)$ that is thought to protect women against sexually transmitted diseases (STDs) and inhibit the proliferation of opportunistic endogenous bacteria $([10,14,15]$, reviewed in [4]). Indeed, the loss of a lactobacillidominated community and subsequent increase in vaginal $\mathrm{pH}$ can lead to the overgrowth of anaerobic bacteria, referred to as bacterial vaginosis (BV), which is associated with infertility, preterm birth, maternal infections, and increased risk of STDs [3, 16-19].

In humans, a variety of endogenous and exogenous factors alter the relative abundance of Lactobacillus and vaginal microbial composition [20, 21]. In particular, estrogen stimulates the proliferation of the vaginal epithelium, increasing available glycogen in the vagina [22, 23]. As such, the bacterial composition of the vaginal microbiome is strongly affected by normal fluctuations in estrogen that occur during puberty and menopause, between reproductive states, and over the menstrual cycle (e.g., [24-26]). In particular, estrogen peaks during ovulation and this peak is linked to high relative abundance of Lactobacillus spp., low microbial diversity, low vaginal $\mathrm{pH}$, and a stable bacterial community $[2,27,28]$. In addition to estrogen, sexual contact and exposure to maternal bacteria during birth may also influence the vaginal microbiome. During sexual contact, transmission of novel bacteria or neutralization of vaginal acidity by seminal fluid may impact the vaginal environment [29-33]. In support, sexual promiscuity is linked to greater instability in vaginal bacteria and increased risk of BV [20,34, 35]. Additionally, close contact with the mother's vaginal canal during birth leads to the vertical transmission of maternal vaginal bacteria to offspring [36-38].

In contrast to humans, the vaginal microbiota of nonhuman primates (NHPs) - at least all NHPs studied to date-have few lactobacilli (typically $<2 \%$ of resident bacteria) and much higher taxonomic diversity than humans [6, 21, 39-42]. Given these differences, it is unclear whether reproductive state, especially states linked to relatively high or low estrogen levels, will also be associated with predictable changes in NHP vaginal microbiota. Similarly, the effects of sexual contact or vertical transmission on NHP vaginal microbiota are not well understood. To address these gaps, we tested the association between reproductive state, ovarian cycle phase, sexual behavior, and vertical transmission and inter-individual differences in vaginal microbial composition in a well-studied population of wild baboons (Papio cynocephalus) living in the Amboseli ecosystem in Kenya. Similar to other NHPs, baboon vaginal microbiota are characterized by low levels of Lactobacillus spp., high microbial diversity, and high vaginal $\mathrm{pH}$ compared to humans [6, 39, 40, 43, 44]. Baboons also exhibit well-documented changes in external genital morphology that indicate female reproductive state and ovarian cycle phase, including pregnancy, postpartum amenorrhea, and four distinct stages of ovarian cycling (including ovulation; Fig. 1) [45-47]. However, like humans, baboons reproduce year-round: when not pregnant or in postpartum amenorrhea, baboons experience $\sim 34$-day ovarian cycles and can conceive at any time of the year [48, 49]. Also similar to humans, estrogen and vaginal glycogen are highest in baboons around ovulation and decrease during anestrus (i.e., menses) [46, 50-52]. Hence, if baboon vaginal microbiota experience dynamics similar to those in humans, we would expect to observe relatively high levels of lactic acid-producing bacteria during the periovulatory period and relatively low levels during low estrogen periods such as anestrus.

Our primary objective was to test the effects of reproductive state and ovarian cycle phase on the microbial composition of the baboon vaginal microbiome in order to understand whether the reproduction-related changes in baboon vaginal microbiota are parallel to those in humans. In addition, we used data on the baboons' sexual contacts and maternal relationships to test for evidence of horizontal and vertical transmission in the vaginal microbiota. We also explored other variables that may shape inter-individual differences in baboon vaginal microbiota, including kinship, age, dominance rank, social group size (as a measure of available sexual partners), and rainfall (which may affect bacterial exposures in the environment). Finally, we measured vaginal $\mathrm{pH}$ in a separate set of subjects from the same population to understand the relationship between reproductive state and vaginal $\mathrm{pH}$. To our knowledge, this data set represents the largest sample size and the greatest sequencing depth of the vaginal microbiota in a wild, non-human mammal to date. Our results improve our understanding of the factors that shape natural variation in the mammalian vaginal microbiome and provide valuable groundwork for further investigation on how vaginal microbiota contributes to host health and disease risk.

\section{Methods}

\section{Study subjects}

Study subjects for microbial analyses were 48 wild, adult female baboons (Papio cynocephalus), living in five 


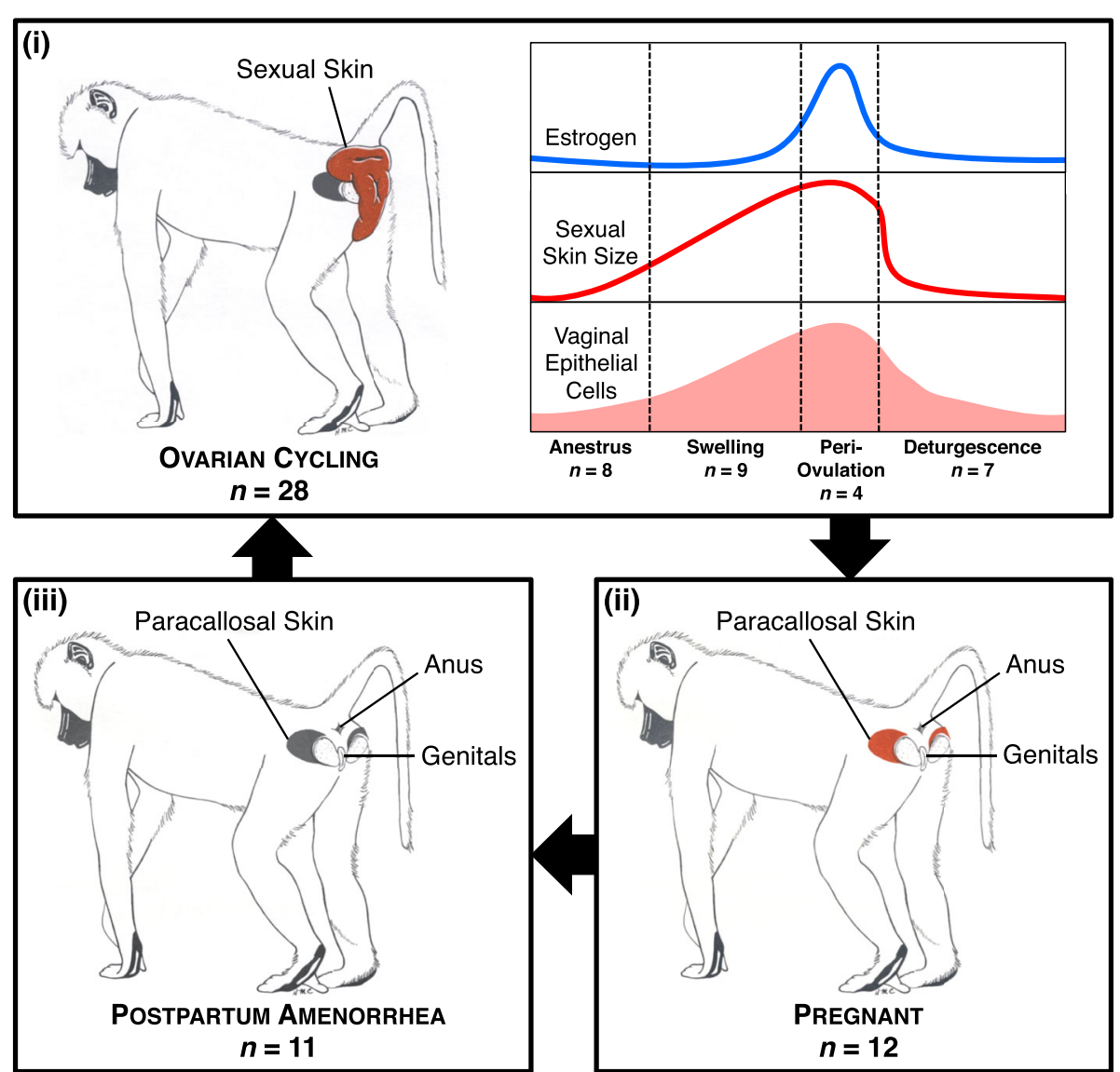

Fig. 1 Schematic representing the progression through reproductive states and ovarian cycle phases in baboons with sample sizes for this study shown for each state/phase. Baboon drawings show the characteristics of the perineal sexual skin and the paracallosal skin associated with each reproductive state. Ovarian cycling includes four phases: (i) swelling ( $n=9$ samples), during which the perineal skin begins to swell; (ii) periovulation ( $n=4$ samples), which occurs at peak swelling, in the 5 days prior to deturgescence; (iii) deturgescence ( $n=7$ samples), when the perineal skin deflates; and (iv) anestrus ( $n=8$ samples), which occurs when the sexual swelling has completely deturgesced (anestrus includes days when females are menstruating). High levels of estrogen during periovulation stimulate the proliferation of the vaginal epithelium, increasing available glycogen in the vagina [22, 23]. If conception occurs during ovarian cycling, females become pregnant $(n=12)$, during which the color of their paracallosal skin changes from dark gray to pink [45]. After birth, the paracallosal skin of females in postpartum amenorrhea (those who either give birth or miscarry, prior to resumption of ovarian cycling; $n=11$ ) gradually returns to dark gray. One sample collected from a female in the process of miscarrying is not included in the sample sizes shown in the figure

different social groups in the Amboseli ecosystem in Kenya. Since 1971, this population has been intensively monitored by the Amboseli Baboon Research Project [53]. All study group members are individually recognized based on morphological characteristics. Experienced observers visit each study group two to three times per week, year-round, during 5-hour monitoring visits. During these visits, observers record information on a wide range of demographic, reproductive, and behavioral events, allowing us to correlate vaginal microbial composition with reproductive status (Fig. 1) and several other host traits, including history of sexual contacts, maternal relationships, kinship, age, dominance rank, social group size, and rainfall. Detailed information on data collection for each of these variables can be found in Additional file 1: Supplemental methods. A table describing all metadata for each sample can be found in Additional file 2: Table S1, with further information on ovarian cycle phase at the time of sample collection in Additional file 3: Figure S1.

\section{Sample collection, 16S rRNA gene sequencing, and sequence processing}

We collected 52 vaginal swabs from 48 females between 2007 and 2010 (four individuals were sampled twice). Samples were collected from sexually mature females (range: 4-26 years; median: 8.5 years) that were anesthetized using an anesthetic bearing dart $[54,55]$. Subjects were chosen opportunistically, but we excluded females in the second half of pregnancy and those with dependent infants (i.e., <270 days old). Once anesthetized, a sterile cotton swab was inserted into the female's 
vaginal canal, gently rotated several times, and placed in $1 \mathrm{ml}$ of RNAlater (Qiagen, Valencia, CA, USA).

Samples were stored at ambient temperature in the field and frozen at $-20{ }^{\circ} \mathrm{C}$ upon arrival in the United States. Bacterial DNA was extracted from the swabs using the PowerSoil DNA Isolation kit (MO BIO Laboratories, Inc., Carlsbad, CA, USA), with modifications to the manufacturer's instructions to accommodate the cotton swabs (see Additional file 1: Supplemental methods). A 102 base pair region of the $16 \mathrm{~S}$ rRNA gene $\mathrm{V} 4$ region was amplified and sequenced using the methods developed by Caporaso et al. [56] with library preparation modifications by Davenport et al. [57]. Samples were multiplexed and sequenced in triplicate on three lanes of two Illumina HiSeq2000 flow cells (CA, USA).

Initial quality filtering was performed using PRINSEQ lite v.0.20.4 [58]. Sequences with ambiguous bases and/ or with mean Phred quality scores $\leq 25$ were discarded. To identify operational taxonomic units (OTUs), we employed open reference OTU picking. Specifically, representative sequences for first-round OTU-picking were chosen using USEARCH v.7.0 [59] with an initial OTU subsample depth of $2 \%$. OTU clustering was completed using the UPARSE algorithm [60], which includes chimera filtering [59]. Sequences that did not match first-round OTUs were clustered and used as reference sequences in second-round OTU-picking. To reduce the risk of including OTUs that were PCR artifacts, all OTUs that occurred in only one sample were removed. Taxonomic identities were assigned to each OTU in QIIME v.1.8.0 [61] using the RDP Classifier v.2.2 [62] with the QIIME-formatted SILVA reference database (release 123, available at https://www.arb-silva.de/down load/archive/qiime/) [63, 64]. Use of Greengenes [65] as the reference database produced qualitatively identical results, but with higher rates of unclassified OTUs. To verify that all sequences were from the $16 \mathrm{~S}$ rRNA gene V4 region, reference sequences were aligned using the Python implementation of the NAST alignment algorithm (PyNAST)[66], and sequences that did not align were removed. To control for differences in sequencing depth between samples, we normalized read counts using cumulative-sum scaling implemented in the $R$ package metagenomeSeq $[67,68]$. This OTU table was used to calculate beta diversity and for differential relative abundance analyses. All analyses were repeated using an alternative OTU table rarefied to $1,777,373$ reads per sample, and there were no qualitative differences in the results (data not shown).

\section{Statistical analyses}

\section{Predictors of alpha diversity}

A full summary of all statistical analyses conducted in $R$ is included in Additional file 4. Using the pre- normalized OTU table, we calculated the number of unique OTUs in a sample (i.e., richness) and Shannon's diversity index, which accounts for the distribution of OTU abundances (Additional file 2: Table S1). To test predictors of microbial alpha diversity, we constructed multivariate linear regression models for both alpha diversity metrics in $R$ (version 3.2.2, R Foundation for Statistical Computing, Vienna, Austria). We modeled the following as fixed effects: sequencing read count (to control for variation in sequencing depth between samples), age, reproductive state or ovarian cycle phase, dominance rank, presence or absence of rainfall in the 30 days prior to sample collection, the number of individuals in a female's social group at sample collection (i.e., social group size), and level of promiscuity, estimated using the average number of consortship partners per ovarian cycle. Consortships are defined as sustained proximity between an adult male and female with a turgescent swelling [69]. Most sexual contact with intromission occurs in the context of consortships [54, 70, 71]. Although four individuals were sampled twice, we report models without individual identity because variation in alpha diversity among samples from the same female did not differ significantly from samples between females (Additional file 3: Figure S2). Furthermore, the inclusion of individual identity as a random effect in our models did not qualitatively change our results. Model selection was performed using stepwise backward regression with the stepAIC function from the $R$ package MASS [72]. An alpha value of 0.05 was used as a threshold for inclusion in the final model.

\section{Predictors of microbial beta diversity}

To identify predictors of vaginal microbial similarity between samples, we performed principal coordinates analyses (PCoAs) and PERMANOVAs using Bray-Curtis dissimilarity and weighted UniFrac distance [73]. Predictor variables in the PERMANOVAs included age, reproductive state or ovarian cycle phase, dominance rank, rainfall, social group size, and level of promiscuity. Again, we did not include individual identity because variation in beta diversity between samples from the same female did not differ significantly from samples between females (Additional file 3: Figure S3).

\section{Testing for horizontal and vertical transmission}

To test for horizontal transmission, we used partial Mantel tests implemented in $R$ package vegan [74] to correlate the extent to which females shared the same male consortship partners over their lifetime (partner sharing) with estimates of vaginal microbial community dissimilarity (i.e., Bray-Curtis and weighted UniFrac). Because similar sexual history between two vaginal samples could be due to the samples being from the same host, 
from hosts living in the same social group, or from hosts of similar age, we separately tested the correlations between microbial dissimilarity and each of the following matrices: a binary matrix of individual identity (i.e., samples from same or different individual), a binary matrix of social group co-residency (i.e., same or different social group), and a matrix of the absolute difference in ages between all pairs of sample dyads. For all partial Mantel tests, we controlled for reproductive state and ovarian cycle phase by including a third, binary matrix that indicated whether pairwise samples were from the same or different reproductive state or ovarian cycle phase. We calculated $P$ values for all partial Mantel tests based on comparison of the observed Pearson correlation coefficient to Pearson coefficients calculated from 10,000 permutations.

To test for vertical transmission from mother to offspring, we used non-parametric Kruskal-Wallis tests to compare vaginal microbial dissimilarity between samples from maternal siblings ( $n=10$ sibling pairs) to the dissimilarity between samples from paternal siblings $(n=15$ sibling pairs) and unrelated pairs of individuals $(n=$ 1049). If vertical transmission plays a strong role in shaping female vaginal microbiota, microbial communities from maternal siblings should be significantly more similar to each other than the communities of both paternal siblings and unrelated dyads. To test the effects of pairwise genetic relatedness beyond motheroffspring effects on vaginal microbiota, we used partial Mantel tests to correlate a pedigree of both maternal and paternal relatedness with pairwise microbial dissimilarity, controlling for reproductive state.

\section{Identifying taxa associated with different reproductive states and ovarian cycle phases}

We used linear discriminant analysis (LDA) effect size (LEfSe; Galaxy v.1.0) to identify taxa that differed significantly in relative abundance between reproductive states and ovarian cycle phases. LEfSe is a method for high-dimensional biomarker discovery that combines standard statistical tests (i.e., the Kruskal-Wallis rank sum test and the pairwise Wilcoxon test) with linear discriminant analysis to detect taxa that explain the most variation between two or more classes [75]. We set the alpha value for the Kruskal-Wallis test at 0.01 and the threshold on the logarithmic LDA score at 3.0.

\section{Determination of vaginal $\mathrm{pH}$}

To understand the relationship between reproductive state and vaginal $\mathrm{pH}$, we measured the vaginal $\mathrm{pH}$ of a separate set of 20 female Amboseli baboons between May and July of 2015 and 2016. None of these individuals were included in the vaginal microbiota portion of this study, and so we did not combine the $\mathrm{pH}$ data with microbial data for any analysis. However, all subjects for microbial and $\mathrm{pH}$ analyses came from the same wild population and lived together in the same five social groups, such that the $\mathrm{pH}$ data we collected should be representative of vaginal tract $\mathrm{pH}$ in our study system (as opposed to measurements from other species, populations, or captive animals). Measurements of $\mathrm{pH}$ were collected following Thoma et al. [24]. Briefly, a pH-Fix paper strip ( $\mathrm{pH}$ 4.5-10.0, Macherey-Nagel, Düren, Germany) was affixed to a sterile pediatric tongue depressor and inserted into the vaginal canal with the aid of a speculum. The tongue depressor was kept in the vagina for $10 \mathrm{~s}$ and then the $\mathrm{pH}$ reading was taken immediately following removal.

\section{Results}

Baboon vaginal microbiota consist of a core set of taxa not dominated by Lactobacillus spp.

We generated a total of $188,665,626$ reads from 48 female baboons ( $n=52$ samples; $1,777,373-7,024,839$ reads per sample; mean =3,628,185; Additional file 2: Table S1). Taxonomic assignments revealed representatives of 29 bacterial and archaeal phyla (Fig. 2a). Of these 29, 11 phyla were found in $100 \%$ (52/52) of samples, six of which had a mean relative abundance greater than 1\%, including: Firmicutes (33\%), Fusobacteria (29\%), Proteobacteria (13\%), Bacteroidetes (11\%), Actinobacteria (10\%), and Tenericutes (3\%) (Fig. 2a; Additional file 2: Table S2). Forty-three genera were also found in all 52 samples, including multiple genera that have been linked to BV in humans, such as Mobiluncus, Atopobium, Prevotella, Mycoplasma, and Sneathia (Fig. 2b; Additional file 2: Table S2)[76].

As found in prior studies (e.g., [6, 43, 44, 77]), the baboon vaginal microbiota had markedly lower levels of lactobacilli than human vaginal microbiota. Lactobacillus spp. occurred in only 85\% (44/52) of baboon samples, as compared to near $100 \%$ prevalence in women (e.g., [1]). Moreover, the median relative abundance of Lactobacillus spp. was only $0.00063 \%$ in baboons (range: 0-0.93\%), compared to a reported median relative abundance of $96 \%$ in humans (range: 0\%-99.9\%) [1]. However, other lactic acid-producing bacteria (LAB), including Streptococcus, Facklamia, Aerococcus, and unclassified members of the order Lactobacillales, were relatively abundant in all baboon samples, with a mean relative abundance of $10 \%( \pm 16 \%$ SD; Fig. 2b; Additional file 2: Table S2). Finally, approximately $12 \%$ of OTUs, comprising approximately $1.5 \%$ of reads, could not be classified beyond the kingdom level, suggesting that a large number of novel taxa colonize the baboon vagina. These unclassified OTUs were unlikely to be sequencing artifacts as all appeared in more than one sample, and the 

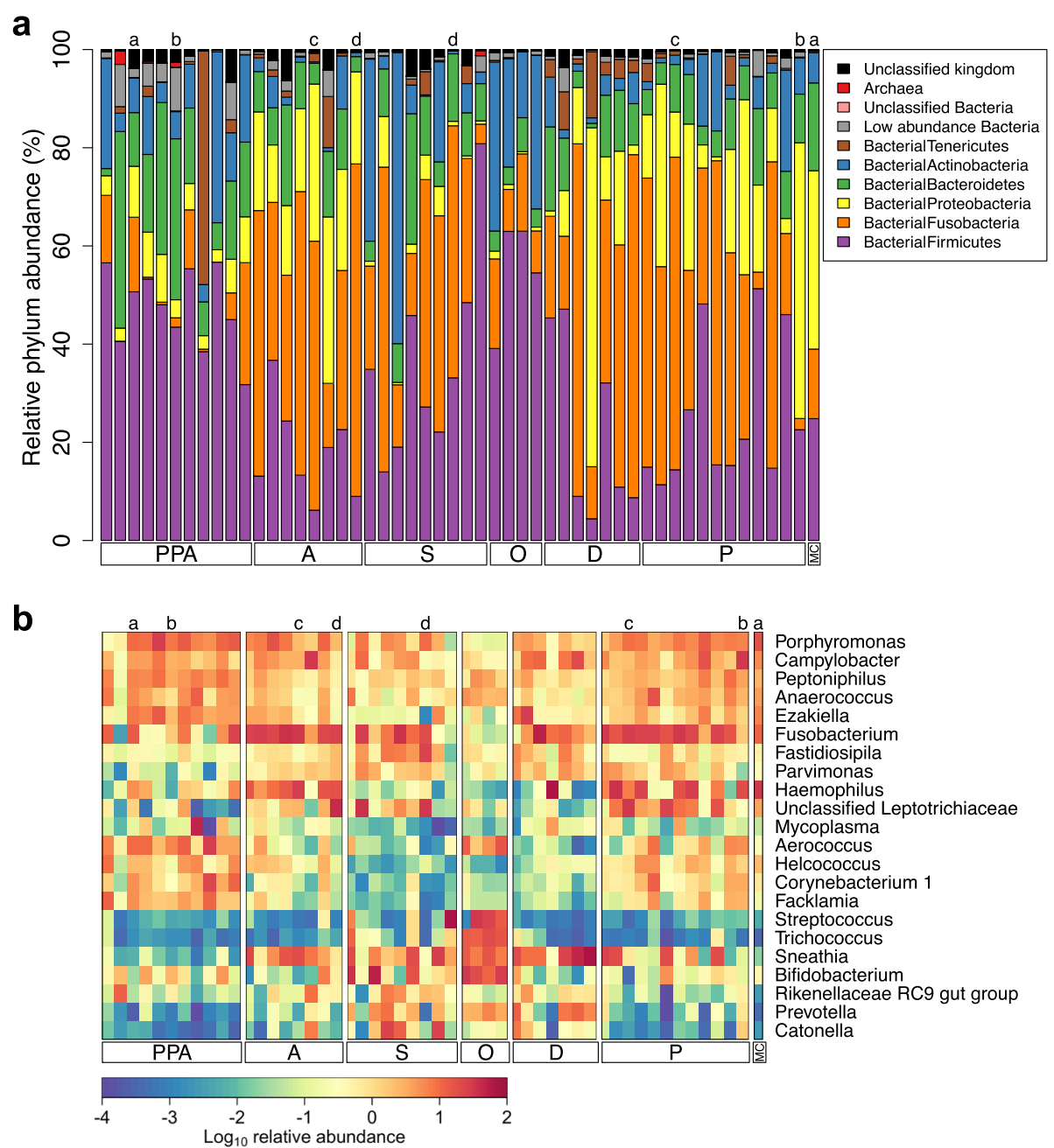

Fig. 2 Relative abundance of bacterial and archaeal (a) phyla and (b) genera in the baboon vaginal microbiota by sample $(n=52)$. In panel a, each bar represents one sample; colors depict the proportion of reads assigned to a particular phylum in each sample. Only phyla with $\geq 1 \%$ mean relative abundance are shown. Phyla present at $<1 \%$ are grouped into the category "Low abundance Bacteria". Panel $\mathbf{b}$ shows a heatmap of the log ${ }_{10}{ }^{-}$ transformed relative abundance of bacterial genera. Only genera with $\geq 1 \%$ mean relative abundance are shown. Each column represents one sample. Genera along the $y$ axis were ordered based on hierarchical clustering by Euclidian distance. For both panels $\mathbf{a}$ and $\mathbf{b}$, samples are ordered based on female reproductive state and ovarian cycle phase (Fig. 1): PPA, postpartum amenorrhea; $A$, anestrus; $S$, swelling; $O$, periovulation; $D$, deturgescence; $P$, pregnant; and $M C$, miscarrying. Matching lower case letters above bars and columns denote samples from the same individual

distribution of their prevalence across samples closely resembled the distribution of classified OTUs.

\section{The composition of vaginal microbiota changes with female reproductive state and ovarian cycle phase Alpha diversity}

Female reproductive state and ovarian cycle phase were strongly correlated with Shannon's diversity, but not with OTU richness (Fig. 3a; Additional file 2: Table S3). Shannon's diversity was lowest in cycling females and highest in females experiencing postpartum amenorrhea (PPA), with some evidence for increasing Shannon's diversity throughout the first half of pregnancy (Fig. 3a; Additional file 2: Table S3; Additional file 3: Figure S4).
Among the 28 females experiencing ovarian cycling (i.e., anestrus, swelling, periovulation, or deturgescence), anestrous females had the highest Shannon's diversity (Fig. 3a; Additional file 2: Table S4), while OTU richness was higher in both anestrous and periovulatory females compared to swelling and deturgescent females (Fig. 3b; Additional file 2: Table S4).

Aside from reproductive state and ovarian cycle phase, we also found that social group size and rainfall in the 30 days prior to sample collection predicted microbial alpha diversity. Females who were members of larger social groups had lower Shannon's diversity, compared to those in smaller groups (Fig. 3c; Additional file 2: Tables S3 and S4). This pattern may be caused by higher 

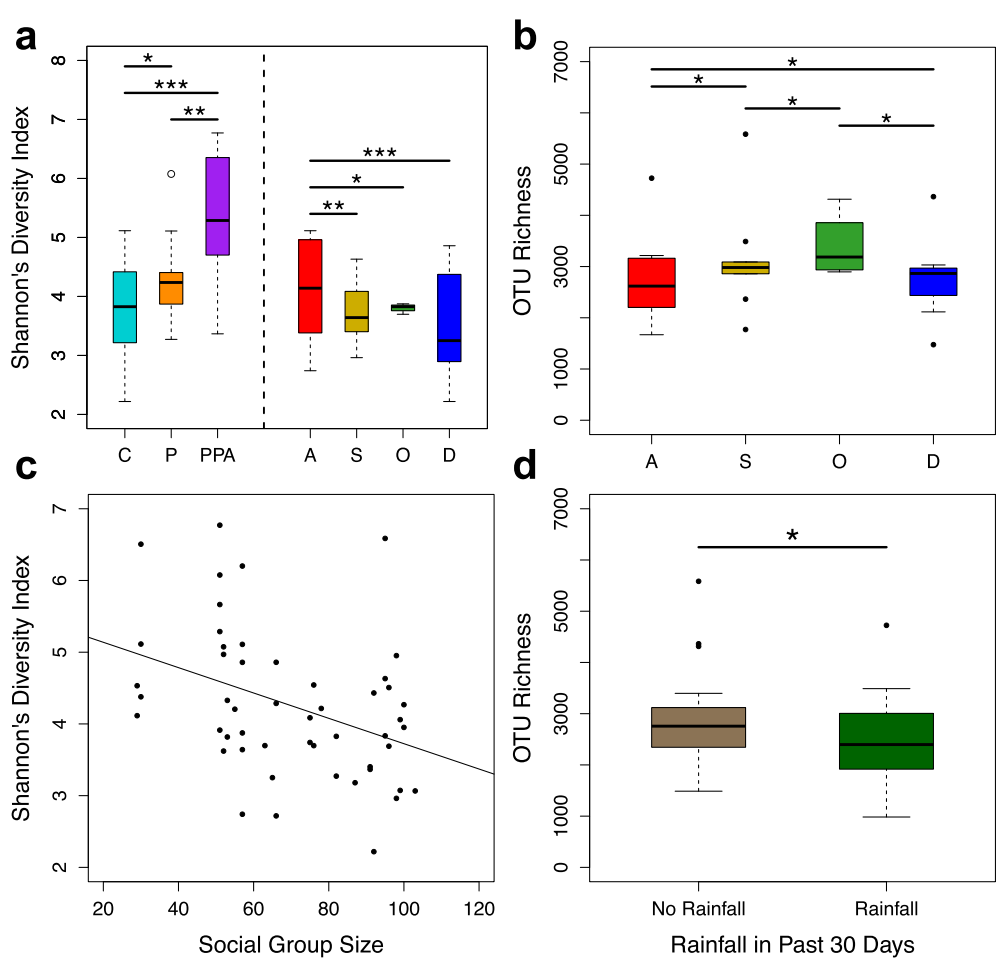

Fig. 3 Plots summarizing major results of best supported multivariate linear regression models predicting Shannon's diversity index and OTU richness (Additional file 3: Tables S3 and S4). a Shannon's diversity index versus reproductive state $(n=51)$ and ovarian cycle phase $(n=28)$. b OTU richness versus ovarian cycle phase (c) Shannon's diversity index versus social group size (d) OTU richness versus rainfall in the previous 30 days. $C$, ovarian cycling; $P$, pregnant; $P P A$, postpartum amenorrhea; $A$, anestrus; $S$, swelling; $O$, periovulation; $D$, deturgescence. ${ }^{*} P \leq 0.05$; ${ }^{* *} P<0.01$; ${ }^{* *} P<0.001$

rates of intromission, and hence more disruption of the vaginal microbiota of females in larger versus smaller social groups. In support, females in larger social groups experienced more consortships and consortship partners per ovarian cycle than females in smaller groups (GLMMs: $\quad$ consortships: $z \quad$ value $=10.17, \quad P<10^{-16}$; consortship partners: $z$ value $=5.47, \quad P=4.62 \times 10^{-8}$; Additional file 3: Figure S5). Additionally, females sampled in rainy months had significantly lower OTU richness than females sampled in dry months, suggesting that the external environment affects vaginal exposure to bacteria (Fig. 3d; Additional file 2: Table S3). No other factors, including female age, dominance rank, or level of promiscuity predicted variation in either alpha diversity metric.

\section{Beta diversity}

Female reproductive state and ovarian cycle phase were also strongly correlated with the composition of vaginal microbiota (Fig. 4; Additional file 3: Figure S6). Indeed, differences in reproductive state explained 18 to $19 \%$ of the variation in overall community composition (Fig. 4a; Additional file 3: Figure S6A; PERMANOVAs: BrayCurtis: Pseudo-F $=5.24, \quad P<10^{-5}$; weighted UniFrac: Pseudo-F $\left.=5.71, \quad P<10^{-5}\right)$. Among the 28 samples collected during ovarian cycling, cycle phase explained 22 to $39 \%$ of the variation in microbial composition (Fig. 4b; Additional file 3: Figure S6B; PERMANOVAs: Bray-Curtis: Pseudo-F $=5.09, P<10^{-5}$; weighted UniFrac: Pseudo-F $=2.31, P=0.0032$ ). Of particular interest, periovulation, as compared to swelling, anestrus, and deturgescence, was associated with a distinct vaginal microbiota, with significant differentiation along both the 1st and 2nd principal coordinate axes of the PCoA plot (green dots in Fig. 4b; Additional file 3: Figure S6B).

\section{Bacterial taxa, including some linked to bacterial vaginosis, vary in their relative abundance across female reproductive states}

Given the striking correlation between reproductive state and community composition, we used LEfSe to identify which taxonomic groups experienced the largest changes in relative abundance when females transition from one reproductive state to another. We first compared females in the ovarian cycling phase to those in the next reproductive phase, pregnancy, and likewise compared females in late PPA to those in ovarian cycling. Of the 29 phyla tested, taxa from 7 phyla (24\%) showed significant changes in relative abundance in at least one of the two reproductive state comparisons $(P \leq 0.01$; Fig. 5a). 

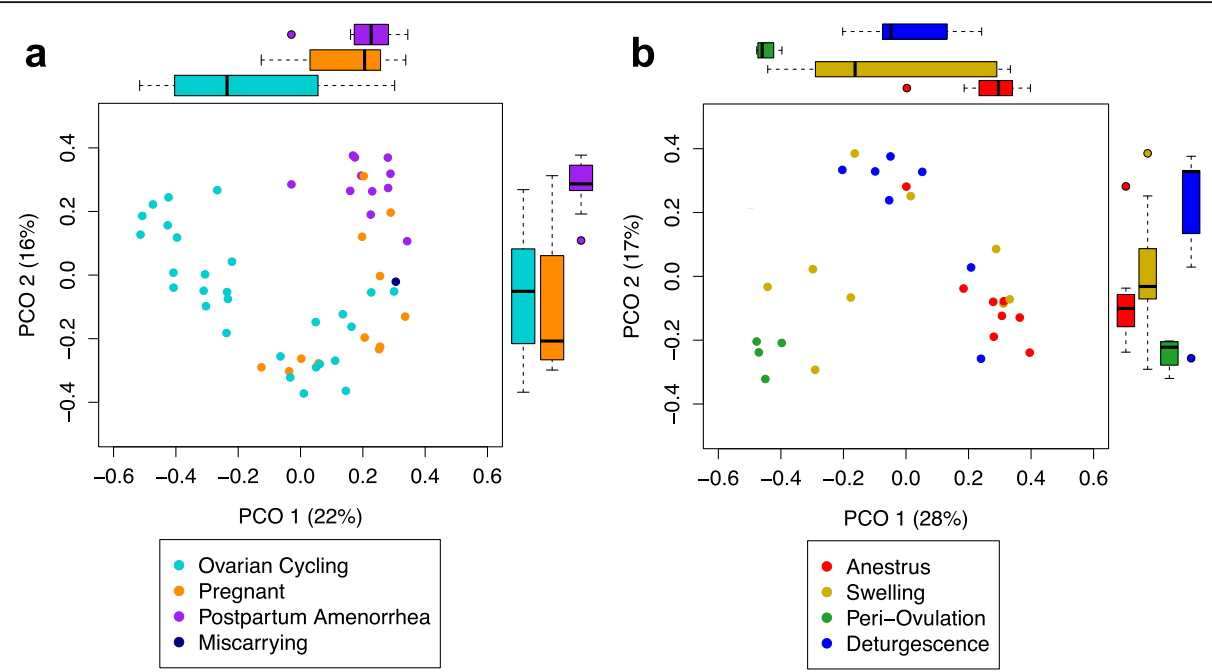

Fig. 4 Principal coordinates plots based on Bray-Curtis dissimilarities for vaginal microbial communities as a function of (a) reproductive state $(n=52)$ and $(\mathbf{b})$ ovarian cycle phase $(n=28)$. Boxplots are included to help visualize differences between reproductive states and ovarian cycle phases across principal coordinates axes 1 and 2

Compared to both pregnant females and those in PPA, cycling individuals had elevated levels of common BVassociated genera, including Sneathia, Prevotella, and Mobiluncus [76]. Conversely, cycling females had relatively low levels of multiple other taxa, including the genus Cornebacterium 1, the LAB family Aerococcaceae-particularly from the genera Facklamia and Aerococcus-and multiple genera from the Clostridiales family XI. Furthermore, there appeared to be a negative relationship between the two dominant phyla Firmicutes and Fusobacteria across reproductive states, with cycling females exhibiting high levels Fusobacteria and low levels of Firmicutes, and PPA females displaying the opposite trend (Fig. 5a).

\section{The periovulatory period is associated with elevated Lactobacillales}

Because community composition varied within the phases of the ovarian cycle (Fig. 4b), we also used LEfSe to identify the microbial taxa that exhibited the greatest changes in relative abundance during periovulation or anestrus relative to other cycle phases. These two phases may exhibit particularly distinct microbial communities because they represent the periods of maximum and minimum estrogen and vaginal glycogen within the ovarian cycle [46, 50-52]. Overall, samples from periovulatory females exhibited significantly higher relative abundance of Bacilli, especially members of the lactic acid-producing order Lactobacillales (mean: $44 \pm 10 \%$ $\mathrm{SD})$, and significantly lower relative abundance of the family Fusobacteriaceae, as compared to the other three cycle phases (Fig. 5b). Indeed, there was a striking tradeoff in relative abundance between Firmicutes and
Fusobacteria throughout the ovarian cycle, with high levels of Firmicutes and low levels of Fusobacteria around ovulation and the opposite pattern at the deturgescence to anestrus transition (Additional file 3: Figure S8). Additionally, compared to swelling, periovulating, and deturgescing females, anestrous individuals had significantly higher levels of genera from the Clostridiales family XI, the phylum Proteobacteria-particularly from the genus Haemophilus - and lower levels of the BVassociated genera Mobiluncus and Prevotella (Fig. 5b).

\section{Vaginal pH fluctuates over the ovarian cycle}

The unique taxonomic composition associated with periovulation, especially the high relative abundance of lactic acid-producing bacteria, suggests that vaginal $\mathrm{pH}$ might be lowest in the time period around ovulation. To test the relationship between reproductive state and vaginal $\mathrm{pH}$, we measured vaginal $\mathrm{pH}$ in a separate set of 20 female baboons living in the same social groups as the original subjects. Vaginal $\mathrm{pH}$ varied significantly across the reproductive states ( $\mathrm{pH}$ range: 5.5-9.0), with the lowest vaginal $\mathrm{pH}$ in females with swollen, turgescent sexual skins, and the highest vaginal $\mathrm{pH}$ in females experiencing pregnancy and postpartum amenorrhea (Kruskal-Wallis test: $H=11.78, P=0.019$; Additional file 3: Figure S8). This finding suggests that vaginal $\mathrm{pH}$ fluctuates across the ovarian cycle, with $\mathrm{pH}$ falling to more acidic levels as females approach ovulation.

\section{Sharing sexual partners predicts vaginal microbial similarity, but vertical transmission does not} While reproductive state and ovarian cycle phase exerted dominant effects on vaginal microbiota, we also tested 


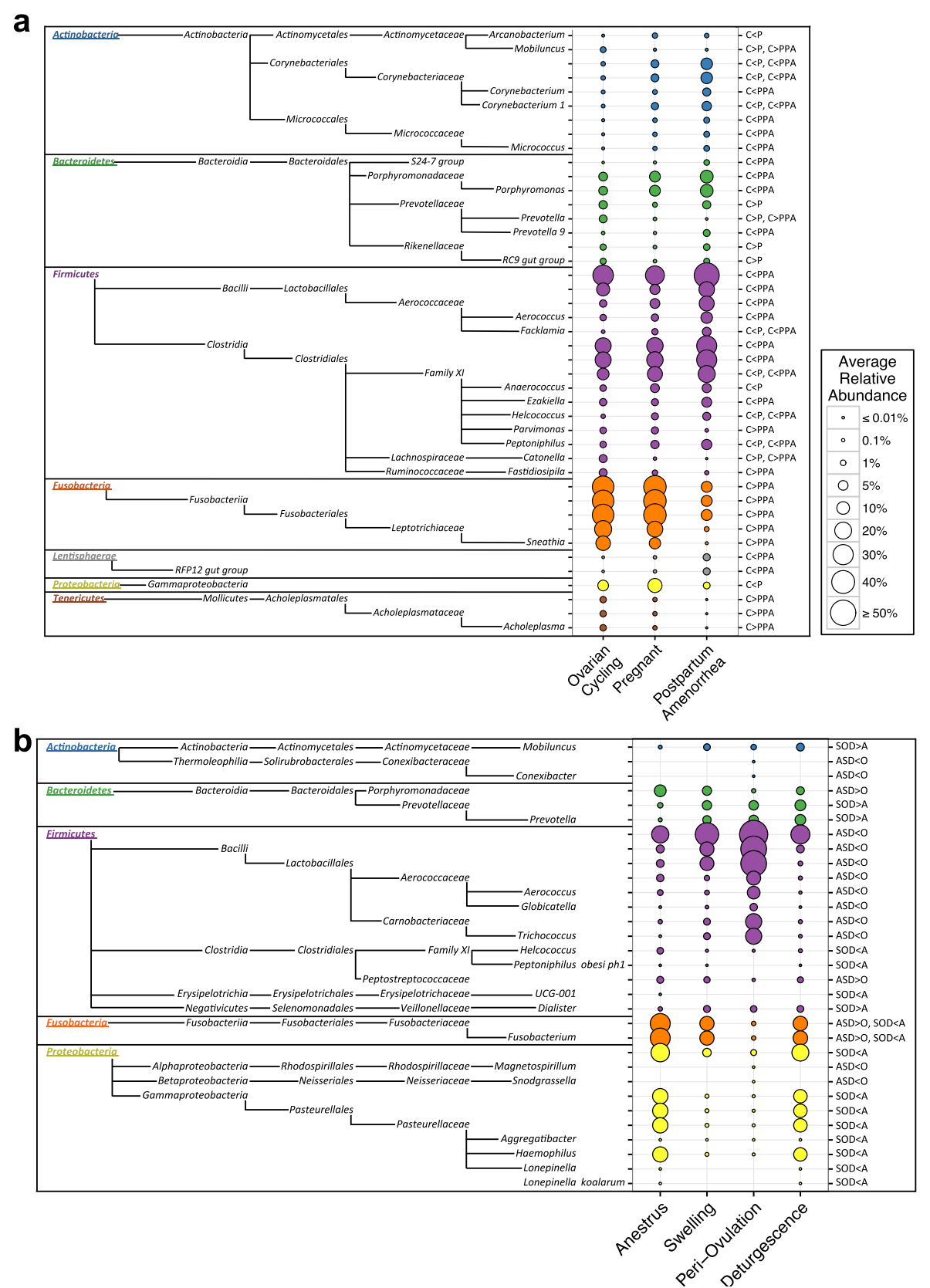

Fig. 5 Bubble plots representing all vaginal bacterial taxa that have significant differences in relative abundance between reproductive states and ovarian cycle phases as identified by LEfSe. Pairwise comparisons included (a) ovarian cycling versus pregnancy (C vs. P), ovarian cycling versus postpartum amenorrhea (C vs. PPA), (b) periovulation versus anestrus, swelling, and deturgescence ( $\mathrm{V}$ vs. ASD), and anestrus versus swelling, periovulation, and deturgescence (A vs. SOD). Circle size is proportional to each taxon's average relative abundance and circle color differentiates between phyla. Significant comparisons $(P \leq 0.01)$ are noted on the right side of the plot

for evidence of horizontal and vertical transmission in shaping vaginal microbial communities. In the case of horizontal transmission, we predicted that females who share the same sexual partners should exhibit more similar vaginal microbiota than females who do not share sexual partners. In support, we found that females with more overlap among partners in their lifetime consortship profiles tended to have slightly more similar vaginal microbial communities, controlling for reproductive state and ovarian cycle phase, than females with less overlap among partners (partial Mantel tests: Bray-Curtis dissimilarity: $r=0.052, P=0.046$; weighted UniFrac: $r=0.043, P=0.076$; Additional file 2: Table S5). This pattern could not be explained by individual identity, similarity in age, or social group co-residency (Additional file 2: Table S5). 
With respect to vertical (mother-offspring) transmission, we predicted that maternal siblings would exhibit more similar vaginal microbiota than paternal siblings or unrelated females. However, we found no evidence that vertical transmission affects the microbial composition of the baboon vaginal cavity in adulthood. Controlling for female reproductive state and ovarian cycle phase, pairwise samples from maternal siblings did not have more similar vaginal microbial communities than samples from paternal siblings or unrelated individuals (Kruskal-Wallis tests: Bray-Curtis dissimilarity: $H=$ $0.25, P=0.88$; weighted UniFrac: $H=0.77, P=0.68)$. We also found no evidence that overall pairwise genetic relatedness between all the female baboons in our data set explained similarity in vaginal microbial communities (partial Mantel tests: Bray-Curtis dissimilarity: $r=$ $0.015, P=0.30$; weighted UniFrac: $r=0.018, P=0.26$ Additional file 2: Table S5).

\section{Discussion}

To date, the majority of research on the vaginal microbiome of non-human primates has used captive animals (e.g., [6, 39, 41-43, 78]). Recently, several authors have called for greater use of wild subjects in studies of the microbiome, in part because of strong effects of captivity on host-associated microbial communities [79-81]. The study described here is, to our knowledge, only the third to investigate vaginal microbiota composition in wild primates; notably, the previous two studies were restricted to small sample sizes and lacked of information on host reproductive state $[6,77]$. Thus, this work represents the most comprehensive investigation of inter-individual variation in the vaginal microbiota in a wild, non-human primate population.

All primates studied to date, in either captive or wild settings, lack the Lactobacillus spp. dominance typically found in human vaginal microbiota, raising questions about whether the forces that shape the human vaginal microbiome are also important in other primates and mammals. Despite low relative abundance of lactobacilli, we found that many of the same predictors of human vaginal microbiota are also important in wild baboons. Specifically, reproductive state and ovarian cycle phase-especially ovulation-were linked to distinct vaginal microbial communities, which may have consequences for functional aspects of the vaginal microbiome. In addition, females with similar sexual histories also had more similar vaginal microbiota. Together, these results suggest that, despite large differences in vaginal community composition, similar forces influence the communities of humans and nonhuman primates, laying important groundwork for further comparative work on how vaginal microbiota contribute to host health and disease risk across primates.

\section{Responses of the vaginal microbiota to host reproductive state and ovarian cycle phase}

In humans, reproductive state (cycling, pregnancy, or postpartum amenorrhea) is the dominant force shaping the vaginal microbiome [26, 82-84]. These effects are primarily attributed to changing levels of estrogen, which affect the abundance of glycogen in vaginal mucus-a key resource for energy metabolism by lactobacilli [85]. Similar to humans, we found that reproductive state is also the primary driver of inter-individual variation in baboon vaginal microbiota, and many of the patterns we observe are consistent with the idea that fluctuations in estrogen and glycogen drive baboon vaginal microbial dynamics. For instance, like humans, glycogen is lowest in baboon vaginal mucus during both pregnancy and postpartum amenorrhea and highest during ovarian cycling [52]. Paralleling this pattern, the family Aerococcaceae, particularly the genus Facklamia, is prolific during pregnancy and PPA in female baboons, but rare during ovarian cycling. Since Aerococcaceae cannot metabolize glycogen directly and variably ferment maltose [86], the low relative abundance during cycling suggests this taxa is being outcompeted by other bacteria, such as Sneathia and Prevotella, that can metabolize glycogen directly and thus may thrive in a glycogen-rich environment (or, conversely, be more easily outcompeted by Facklamia in a glycogen-poor environment) $[87,88]$.

Within cycling female baboons, estrogen and vaginal glycogen fluctuate across ovarian cycle phases, with the highest levels around ovulation and lowest during anestrus $[46,50-52]$. We find that the relative abundance of lactic acid-producing bacteria (LAB) follows a similar pattern, with an increase around ovulation and decrease during anestrus. Such changes are expected to reduce lactic acid production, and indeed, in baboons, we observe lower vaginal $\mathrm{pH}$ during the swelling phase of the ovarian cycle compared to anestrus. Similar patterns of vaginal $\mathrm{pH}$ have been observed over the human menstrual cycle, with a drop in $\mathrm{pH}$ during the follicular phase, and an increase during menses [27, 89].

It is worth noting that our findings differ from a prior study on the vaginal microbiota of cycling female baboons [43]. In contrast to our results, Uchihashi et al. [43] found no differences between females experiencing different ovarian cycle phases. There are two possible reasons for this finding. First, Uchihashi et al. studied captive baboons, and prior work has found that hostassociated microbial communities can be altered by captivity [40]. Second, and probably more importantly, Uchihashi et al. did not make fine-scaled distinctions between different female ovarian cycle phases. For instance, Uchihashi et al. combined swelling, periovulatory, and deturgescence phases into a single category 
("cycling") and separated menstruating females from those that we termed anestrus (i.e., "non-cycling"). When we reanalyze our data based on Uchihashi et al.'s categories, we still observe significant differences in community composition between cycling and non-cycling females, but there were no significant differences in community composition between menstruating and nonmenstruating females. Combining across cycle phases is common in the literature because it can be challenging to assign female mammals to the correct ovarian cycle phase. However, our results highlight the importance of these characterizations for understanding variation in the vaginal microbiota.

\section{The role of lactic acid-producing bacteria during ovulation}

Consistent with previous work, we find that Lactobacillus is rare in the baboon vaginal microbiota, especially compared to humans $[6,39,40,43,44]$. From a functional standpoint, aspects of human physiology may make the human vagina more lactobacilli-friendly than other animals. For example, humans have significantly higher glycogen and lactic acid levels in vaginal fluid compared to macaques [90]. From an evolutionary perspective, differences in the vaginal microbiome between humans and NHPs may have arisen as a result of variation in sexual behavior and disease risk $[6,21]$. Specifically, continual sexual receptivity and long intromission may expose humans to more pathogens (particularly STDs) compared to NHPs, leading to selection for a lactobacilli-dominated community $[6,21]$. By extension, since STDs are not unique to humans, one might predict that NHPs should have the most protective microbial community when sexual activity and disease risk are greatest [91, 92]. For example, in baboons, the majority of sexual contact takes place within the second half of the swelling phase and periovulation [48]. Correspondingly, we observe the highest levels of LAB during this period, and find that BVrelated bacteria are abundant during the periovulatory period, indicating that ovulation may be a time of high disease risk. In a separate data set, we also observe the lowest vaginal $\mathrm{pH}$ near this time; however, even the lowest vaginal $\mathrm{pH}$ measurements we recorded $(\mathrm{pH}=5.5)$ were higher than what is considered protective and healthy in human women, whose vaginal $\mathrm{pH}$ typically ranges from 3.5 to $5.0[1,11,85]$. This suggests that defense mechanisms other than $\mathrm{pH}$ may also be at work in baboons, such as competitive exclusion, production of bacteriocins and other antimicrobial compounds [93, 94], and microbial interaction with the host immune system [95].

In addition to a possible protective function, the unique microbial community associated with the periovulatory phase may also have implications for sexual attraction. While male baboons typically identify sexually receptive females based on visual signals, such as sexual swellings [48], there is debate about how males choose one ovulating female over another (e.g., [96]). Vaginal microbiota may generate unique olfactory cues that signal ovulation, female 'quality', or likelihood of conception [97]. Indeed, work in a wide range of animals, including humans, suggests that many bacteria produce volatile compounds that can convey information about their hosts [98, 99]. For example, in humans, levels of volatile organic acids, including lactic acid, change over the human menstrual cycle [2], which may influence the attractiveness of female vaginal secretions [100].

\section{Transmission of vaginal bacteria}

Past work in a wide range of animals indicates that microbial transmission occurs between sexual partners and from mother to offspring during birth [92, 101, 102]. While we find no evidence that vertical transmission exerts long-term effects on the vaginal microbiota of adult female baboons, our results do suggest that sexual contact leads to horizontal transmission of baboon vaginal bacteria. It is well established that copulation facilitates the spread of pathogens, and that multiple sexual partners can further increase the risk of infection [103, 104]. However, recent work suggests that commensal, and even beneficial, bacteria are also being transmitted between partners during sexual contact [102]. Thus, more promiscuous mating systems may promote exposure to these beneficial microbes [105]. In humans, there is some evidence for the sharing of commensal bacteria between sexual partners [30, 31], but, to our knowledge, our study provides the first evidence in a non-human primate that sharing sexual partners can result in similar commensal microbial communities. Interestingly, our results also suggest that an increased number of sexual partners may perturb the vaginal microbiota and reduce microbial alpha diversity. Indeed, in humans, promiscuity has been linked to greater instability in vaginal bacteria [20] and BV [106, 107]. However, it is yet to be determined whether these changes are due to the introduction of novel bacteria or neutralization of the vaginal environment due to repeated insemination [29].

\section{Conclusions}

Like other non-human primates, baboons lack the Lactobacillus spp. dominance typically found in the human vaginal microbiome. Despite this difference and similar to humans, we found that reproductive state was the strongest predictor of the baboon vaginal microbiota, with effects on both microbial composition and $\mathrm{pH}$. These results highlight the importance of accounting for fine-scale differences in reproductive state and emphasize the need for future studies to include this information whenever possible. Moreover, we 
found that during ovulation, the baboon vaginal microbiota exhibited traits reminiscent of those observed in humans, which are believed to defend against pathogens. This included the highest levels of lactic acidproducing bacteria, which predicts the lowest vaginal $\mathrm{pH}$. Overall, our findings suggest that the vaginal microbiome of humans and baboons are under similar selective forces, which indicates these forces may be fundamentally important to many mammals. However, the unique nature of the human vaginal microbiome suggests that other factors shape vaginal microbial communities in humans. Future work should focus on disentangling the evolutionary pressures common to all mammals and those that are unique to humans.

\section{Additional files}

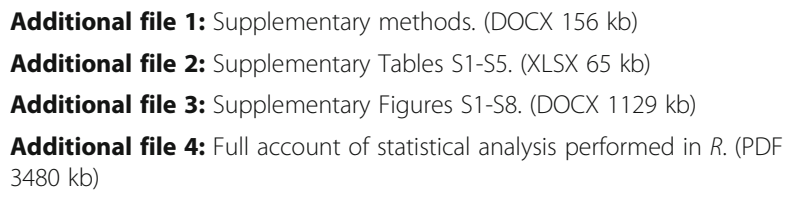

\section{Abbreviations}

A: Anestrus; BV: Bacterial vaginosis; C: Ovarian cycling; D: Deturgescence; LAB: Lactic acid-producing bacteria; LDA: Linear discriminant analysis; LEfSe: Linear discriminant analysis effect size; NHP: Non-human primate; O: Periovulation; OTU: Operational taxonomic unit; P: Pregnant; PCOA: Principal coordinates analysis; PPA: Postpartum amenorrhea; S: Swelling; STD: Sexually transmitted disease

\section{Acknowledgements}

We thank the Kenya Wildlife Service, Institute of Primate Research, National Museums of Kenya, National Commission for Science Technology and Innovation, members of the Amboseli-Longido pastoralist communities, Tortilis Camp, and Ker \& Downey Safaris for their assistance in Kenya. Particular thanks to R. Mututua, S. Sayialel, J. Warutere, V. Somen, and T. Wango in Kenya, and J. Altmann, K. Pinc, N. Learn, P. Onyango, and J. Gordon in the US. We also thank S. Morrow for lab assistance, M. Clark, D. Jansen, and L. Grieneisen for help with the data processing pipeline and statistical analyses, and M. Akinyi and A. Lea for assistance with the vaginal $\mathrm{pH}$ measurements.

\section{Funding}

This work was supported by the National Science Foundation Graduate Research Fellowship (NSF DGE-1313583). Support for the Amboseli Baboon Research Project was provided by the National Science Foundation (most recently IOS 1053461 to EAA and DEB 0919200 to SCA) and the National Institute of Aging (R01 AG034513 and P01 AG031719 to SCA).

\section{Availability of data and materials}

Raw sequencing files and associated metadata have been deposited in NCBI's Sequence Read Archive (SRA Accession: SRP076531), https:// www.ncbi.nlm.nih.gov/sra. Sample metadata are uploaded as an additional file associated with this manuscript (Additional file 2: Table S1) and will also be made available on Dryad, http://datadryad.org/. The unrarefied OTU table with taxonomic classifications will also be made available on Dryad.

\section{Authors' contributions}

$E A, J T$, and EM conceived and designed the research. EM performed the laboratory experiments. EM, JL, EA, and JT analyzed the data. JT, EA, and SA provided samples, reagents, and metadata. EM and EA wrote the manuscript with input from all authors. All authors read and approved the final manuscript.

\section{Competing interests}

The authors declare that they have no competing interests.

Consent for publication

Not applicable.

\section{Ethics approval}

All protocols adhered to the laws and guidelines of Kenya (Kenya Research Permit numbers NCOSTI/P/14/2921/3128 to EAA, NCST/RCD/12B/012/57 to

JT, and NCST 5/12B/012/55 to SCA). All protocols were approved by the Animal Care and Use Committees at the University of Notre Dame

(13-11-1352), Duke University (A0840903), and Princeton University (1689).

\section{Author details}

${ }^{1}$ Department of Biological Sciences, University of Notre Dame, Notre Dame, IN, USA. ${ }^{2}$ Institute of Primate Research, National Museums of Kenya, Nairobi, Kenya. ${ }^{3}$ Department of Evolutionary Anthropology, Duke University, Durham, NC, USA. ${ }^{4}$ Duke Population Research Institute, Duke University, Durham, NC, USA. ${ }^{5}$ Department of Biology, Duke University, Durham, NC, USA.

Received: 23 May 2016 Accepted: 4 January 2017

Published online: 19 January 2017

\section{References}

1. Ravel J, Gajer P, Abdo Z, Schneider G, Koenig S, Mcculle S, et al. Vaginal microbiome of reproductive-age women. Proc Natl Acad Sci U S A. 2011; 108:4680-7.

2. Gajer P, Brotman R, Bai G, Sakamoto J, Schuette U, Zhong X, et al. Temporal dynamics of the human vaginal microbiota. Sci Transl Med. 2012;4(132):1-12.

3. Van Oostrum N, De Sutter P, Meys J, Verstraelen H. Risks associated with bacterial vaginosis in infertility patients: a systematic review and metaanalysis. Hum Reprod. 2013;28(7):1809-15.

4. Brotman R. Vaginal microbiome and sexually transmitted infections: an epidemiologic perspective. J Clin Invest. 2011;121(12):4610-7.

5. White B, Creedon D, Nelson K, Wilson B. The vaginal microbiome in health and disease. Trends Endocrin Met. 2011:22(10):389-93.

6. Yildirim S, Yeoman C, Janga S, Thomas S, Ho M, Leigh S, et al. Primate vaginal microbiomes exhibit species specificity without universal Lactobacillus dominance. Int Society Microbial Ecol. 2014;8(12):2431-44.

7. Boskey E, Cone R, Whaley K, Moench T. Origins of vaginal acidity: high D/L lactate ratio is consistent with bacteria being the primary source. Hum Reprod. 2001;16(9):1809-13.

8. Boskey E, Telsch K, Whaley K, Moench T, Cone R. Acid production by vaginal flora in vitro is consistent with the rate and extent of vaginal acidification. Infect Immun. 1999:67(10):5170-5.

9. Mirmonsef P, Hotton A, Gilbert D, Burgad D, Landay A, Weber K, et al. Free glycogen in vaginal fluids is associated with Lactobacillus colonization and low vaginal pH. Plos One. 2014;9(7):1-11.

10. O'hanlon D, Moench T, Cone R. In vaginal fluid, bacteria associated with bacterial vaginosis can be suppressed with lactic acid but not hydrogen peroxide. BMC Infect Dis. 2011;11:1-8

11. O'hanlon D, Moench T, Cone R. Vaginal $\mathrm{pH}$ and microbicidal lactic acid when Lactobacilli dominate the microbiota. Plos One. 2013;8(11):1-8.

12. Spear G, Mckenna M, Landay A, Makinde H, Hamaker B, French A, et al. Effect of $\mathrm{pH}$ on cleavage of glycogen by vaginal enzymes. Plos One. 2015; 10(7):1-10.

13. Spear G, French A, Gilbert D, Zariffard M, Mirmonsef P, Sullivan T, et al. Human alpha-amylase present in lower genital tract mucosal fluid processes glycogen to support vaginal colonization by Lactobacillus. J Infect Dis. 2014; 210(7):1019-28.

14. Nunn K, Wang Y, Dimple H, Humphrys M, Ma B, Cone R, et al. Enhanced trapping of HIV-1 by human cervicovaginal mucus is associated with Lactobacillus crispatus-dominant microbiota. Mbio. 2015;6(5):1-9.

15. Brotman R, Klebanoff M, Nansel T, Yu K, Andrews W, Zhang J, et al. Bacterial vaginosis assessed by gram stain and diminished colonization resistance to incident gonococcal, chlamydial, and trichomonal genital infection. J Infect Dis. 2010;202(12):1907-15.

16. Cherpes T, Meyn L, Krohn M, Lurie J, Hillier S. Association between acquisition of herpes simplex virus type 2 in women and bacterial vaginosis. Clin Infect Dis. 2003;37(3):319-25. 
17. Digiulio D, Callahan B, Mcmurdie P, Costello E, Lyell D, Robaczewska A, et al. Temporal and spatial variation of the human microbiota during pregnancy. Proc Natl Acad Sci U S A. 2015;112(35):11060-5.

18. Atashili J, Poole C, Ndumbe P, Adimora A, Smith J. Bacterial vaginosis and HIV acquisition: a meta-analysis of published studies. AIDS. 2008;22(12): 1493-501.

19. Leitich $\mathrm{H}$, Kiss $\mathrm{H}$. Asymptomatic bacterial vaginosis and intermediate flora as risk factors for adverse pregnancy outcome. Best Pract Res Cl Ob. 2007;21(3): 375-90.

20. Schwebke J, Richey C, Weiss H. Correlation of behaviors with microbiological changes in vaginal flora. J Infect Dis. 1999;180(5):1632-6.

21. Stumpf R, Wilson B, Rivera A, Yildirim S, Yeoman C, Polk J, et al. The primate vaginal microbiome: comparative context and implications for human health and disease. Am J Phys Anthropol. 2013;152:119-34.

22. Ayre $\mathbf{W}$. The glycogen-estrogen relationship in the vaginal tract. J Clin Endocrinol. 1951;11(1):103-10.

23. Patton D, Thwin S, Meier A, Hooton T, Stapleton A, Eschenbach D. Epithelial cell layer thickness and immune cell populations in the normal human vagina at different stages of the menstrual cycle. Am J Obstet Gynecol. 2000;183(4):967-73.

24. Thoma M, Gray R, Kiwanuka N, Aluma S, Wang M, Sewankambo N, et al. Longitudinal changes in vaginal microbiota composition assessed by Gram stain among never sexually active pre- and postmenarcheal adolescents in Rakai, Uganda. J Pediatr Adol Gynec. 2011;24(1):42-7.

25. Cauci S, Driussi S, De Santo D, Penacchioni $P$, lannicelli T, Lanzafame $P$, et al. Prevalence of bacterial vaginosis and vaginal flora changes in peri- and postmenopausal women. J Clin Microbiol. 2002;40(6):2147-52.

26. Macintyre D, Chandiramani M, Lee Y, Kindinger L, Smith A, Angelopoulos N, et al. The vaginal microbiome during pregnancy and the postpartum period in a European population. Sci Rep-Uk. 2015;5:1-9.

27. Wagner G, Ottesen B. Vaginal physiology during menstruation. Ann Intern Med. 1982;96(6):921-3.

28. Wilson J, Lee R, Balen A, Rutherford A. Bacterial vaginal flora in relation to changing oestrogen levels. Int J STD AIDS. 2007;18(5):308-11.

29. Tevi-Benissan C, Belec L, Levy M, Schneider-Fauveau V, Mohamed A, Hallouin $\mathrm{M}$, et al. In vivo semen-associated $\mathrm{pH}$ neutralization of cervicovaginal secretions. Clin Diagn Lab Immun. 1997;4(3):367-74.

30. Liu C, Hungate B, Tobian A, Ravel J, Prodger J, Serwadda D, et al. Penile microbiota and female partner bacterial vaginosis in Rakai, Uganda. Mbio. 2015;6(3):1-9.

31. Eren A, Zozaya M, Taylor C, Dowd S, Martin D, Ferris M. Exploring the diversity of Gardnerella vaginalis in the genitourinary tract microbiota of monogamous couples through subtle nucleotide variation. Plos One. 2011;6(10):1-8.

32. Marrazzo J, Antonio M, Agnew K, Hillier S. Distribution of genital Lactobacillus strains shared by female sex partners. J Infect Dis. 2009; 199(5):680-3.

33. Zozaya M, Ferris $M$, Siren J, Lillis $R$, Myers $L$, Nsuami $M$, et al. Bacterial communities in penile skin, male urethra, and vaginas of heterosexual couples with and without bacterial vaginosis. Microbiome. 2016;4(16):1-10.

34. Koumans E, Sternberg M, Bruce C, Mcquillan G, Kendrick J, Sutton M, et al. The prevalence of bacterial vaginosis in the United States, 2001-2004; associations with symptoms, sexual behaviors, and reproductive health. Sex Transm Dis. 2007;34(11):864-9.

35. Yen S, Shafer M, Moncada J, Campbell C, Flinn S, Boyer C. Bacterial vaginosis in sexually experienced and non-sexually experienced young women entering the military. Obstet Gynecol. 2003;102(5):927-33.

36. Mandar R, Mikelsaar M. Transmission of mother's microflora to the newborn at birth. Biol Neonate. 1996;69(1):30-5.

37. Biasucci G, Rubini M, Riboni S, Morelli L, Bessi E, Retetangos C. Mode of delivery affects the bacterial community in the newborn gut. Early Hum Dev. 2010;86(1):S13-S5.

38. Dominguez-Bello M, Costello E, Contreras M, Magris M, Hidalgo G, Fierer N, et al. Delivery mode shapes the acquisition and structure of the initial microbiota across multiple body habitats in newborns. Proc Natl Acad Sci U S A. 2010;107(26):11971-5.

39. Hashway S, Bergin I, Bassis C, Uchihashi M, Schmidt K, Young V, et al. Impact of a hormone-releasing intrauterine system on the vaginal microbiome: a prospective baboon model. J Med Primatol. 2014;43(2):89-99.

40. Rivera A, Frank J, Stumpf R, Salyers A, Wilson B, Olsen G, et al. Differences between the normal vaginal bacterial community of baboons and that of humans. Am J Primatol. 2011;73(2):119-26.
41. Gravett M, Jin L, Pavlova S, Tao L. Lactobacillus and Pediococcus species richness and relative abundance in the vagina of rhesus monkeys (Macaca mulatta). J Med Primatol. 2012;41(3):183-90.

42. Spear G, Gilbert D, Sikaroodi M, Doyle L, Green L, Gillevet P, et al. Identification of rhesus macaque genital microbiota by 165 pyrosequencing shows similarities to human bacterial vaginosis: implications for use as an animal model for HIV vaginal infection. AIDS Res Hum Retrov. 2010;26(2): 193-200.

43. Uchihashi M, Bergin I, Bassis C, Hashway S, Chai D, Bell J. Influence of age, reproductive cycling status, and menstruation on the vaginal microbiome in baboons (Papio anubis). Am J Primatol. 2015;77(5):563-78.

44. Schlabritz-Loutsevitch N, Gygax S, Dick Jr E, Smith W, Snider C, Hubbard G, et al. Vaginal dysbiosis from an evolutionary perspective. Sci Rep. 2016:6:1-7.

45. Altmann S. The pregnancy sign in savannah baboons. J Zoo Animal Med. 1973;4(2):8-12.

46. Wildt D, Doyle L, Stone S, Harrison R. Correlation of perineal swelling with serum ovarian hormone levels, vaginal cytology, and ovarian follicular development during the baboon reproductive cycle. Primates. 1977:18(2):261-70

47. Shaikh A, Celaya C, Gomez I, Shaikh S. Temporal relationship of hormonal peaks to ovulation and sex skin deturgescence in the baboon. Primates. 1982;23(3):444-52

48. Gesquiere L, Wango E, Alberts S, Altmann J. Mechanisms of sexual selection: sexual swellings and estrogen concentrations as fertility indicators and cues for male consort decisions in wild baboons. Horm Behav. 2007:51(1):114-25.

49. Alberts S, Hollister-Smith J, Mututua R, Sayialel S, Muruthi P, Warutere J, et al. Seasonality and long term change in a savannah environment. In: Brockman D, Van Schaik C, editors. Seasonality In Primates: Studies Of Living And Extinct Human And Non-Human Primates. Cambridge: Cambridge University Press; 2005. p. 157-95.

50. Maclennan A, Wynn R. Menstrual cycle of the baboon. I. clinical features, vaginal cytology and endometrial histology. Obstet Gynecol. 1971;38(3):350-8.

51. Stevens V, Sparks S, Powell J. Levels of estrogens, progestogens, and luteinizing hormone during the menstrual cycle of the baboon. Endocrinology. 1970;87(4):658-66

52. Nyachieo A, Kiulia N, Arimi M, Chai D, Mwenda J. Vaginal histological changes of the baboon during the normal menstrual cycle and pregnancy. East Afr Med J. 2009:86(4):166-72.

53. Alberts $S$, Altmann J. The Amboseli baboon research project: 40 years of continuity and change. In: Kappeler P, Watts D, editors. Long-Term Field Studies Of Primates. Berlin: Springer; 2012. p. 261-87.

54. Altmann J, Alberts S, Haines S, Dubach J, Muruthi P, Coote T, et al. Behavior predicts genetic structure in a wild primate group. Proc Natl Acad Sci U S A 1996;93(12):5797-801.

55. Tung J, Akinyi M, Mutura S, Altmann J, Wray G, Alberts S. Allele-specific gene expression in a wild nonhuman primate population. Mol Ecol. 2011; 20(4):725-39.

56. Caporaso J, Lauber C, Walters W, Berg-Lyons D, Lozupone C, Turnbaugh P, et al. Global patterns of $16 \mathrm{~s}$ rRNA diversity at a depth of millions of sequences per sample. Proc Natl Acad Sci U S A. 2010;108:4516-22.

57. Davenport Er, Mizrahi-Man O, Michelini K, Barreiro Lb, Ober C, Gilad Y. Seasonal variation in human gut microbiome composition. Plos One. 2014; 9(3):1-10.

58. Schmieder R, Edwards R. Quality control and preprocessing of metagenomic datasets. Bioinformatics. 2011:27(6):863-4.

59. Edgar R. Search and clustering orders of magnitude faster than BLAST. Bioinformatics. 2010;26(19):2460-1.

60. Edgar R. Uparse: highly accurate OTU sequences from microbial amplicon reads. Nat Methods. 2013;10(10):996-8.

61. Caporaso J, Kuczynski J, Stombaugh J, Bittinger K, Bushman F, Costello E, et al. QIIME allows analysis of high-throughput community sequencing data. Nat Methods. 2010:7(5):335-6.

62. Wang Q, Garrity G, Tiedje J, Cole J. Naive Bayesian classifier for rapid assignment of rRNA sequences into the new bacterial taxonomy. Appl Environ Microb. 2007;73(16):5261-7

63. Quast C, Pruesse E, Yilmaz P, Gerken J, Schweer T, Yarza P, et al. The SILVA ribosomal RNA gene database project: improved data processing and webbased tools. Nucleic Acids Res, 2013:41(D1):D590-D6.

64. Yilmaz P, Parfrey L, Yarza P, Gerken J, Pruesse E, Quast C, et al. The SILVA and "All-species Living Tree Project (LTP)" taxonomic frameworks. Nucleic Acids Res. 2014;42(D1):D643-D8. 
65. Desantis T, Hugenholtz P, Larsen N, Rojas M, Brodie E, Keller K, et al. Greengenes, a chimera-checked $16 \mathrm{~S}$ rRNA gene database and workbench compatible with ARB. Appl Environ Microb. 2006;72(7):5069-72.

66. Caporaso J, Bittinger K, Bushman F, Desantis T, Andersen G, Knight R. PyNAST: a flexible tool for aligning sequences to a template alignment. Bioinformatics. 2010;26(2):266-7.

67. Paulson J, Stine O, Bravo H, Pop M. Differential abundance analysis for microbial marker-gene surveys. Nat Methods. 2013;10(12):1200-2.

68. Mcmurdie Pj, Holmes S. Waste not, want not: why rarefying microbiome data is inadmissible. Plos Comput Biol. 2014;10(4):1-12.

69. Noë R, Sluijter A. Reproductive tactics of male savanna baboons. Behaviour. 1990;113:117-70.

70. Alberts S, Watts H, Altmann J. Queuing and queue-jumping: long-term patterns of reproductive skew in male savannah baboons, Papio cynocephalus. Anim Behav. 2003;65:821-40.

71. Alberts S, Buchan J, Altmann J. Sexual selection in wild baboons: from mating opportunities to paternity success. Anim Behav. 2006;72:1177-96.

72. Venables W, Ripley B. Modern applied statistics with S. 4th ed. New York: Springer; 2002.

73. Lozupone C, Knight R. UniFrac: a new phylogenetic method for comparing microbial communities. Appl Environ Microb. 2005;71(12):8228-35.

74. Oksanen J, Blanchet FG, Kindt R, Legendre P, O'Hara RB, Simpson GL, Solymos P, Stevens MHH, Wagner H. vegan. 2011, Community Ecology Package. http://cran.r-project.org/web/packages/vegan/index.html.

75. Segata N, Izard J, Waldron L, Gevers D, Miropolsky L, Garrett W, et al. Metagenomic biomarker discovery and explanation. Genome Biol. 2011;12(6):1-18.

76. Onderdonk A, Delaney M, Fichorova R. The human microbiome during bacterial vaginosis. Clin Microbiol Rev. 2016;29(1):223-38.

77. Rivera A, Stumpf R, Wilson B, Leigh S, Salyers A. Baboon vaginal microbiota: an overlooked aspect of primate physiology. Am J Primatol. 2010;72(6):467-74.

78. Spear G, Kersh E, Guenthner P, Vishwanathan S, Gilbert D, Zariffard M, et al. Longitudinal assessment of pigtailed macaque lower genital tract microbiota by 454-sequencing reveals dissimilarity to the genital microbiota of healthy humans. AIDS Res Hum Retrov. 2012;28(10):1244-9.

79. Clayton J, Vangay P, Huang H, Ward T, Hillmann B, Al-Ghalith G, et al. Captivity humanizes the primate microbiome. Proc Natl Acad Sci U S A. 2016;113(37):10376-81.

80. Servick K. Of mice and microbes. Science. 2016;353(6301):741-3.

81. Amato K. Co-evolution in context: the importance of studying gut microbiomes in wild animals. Microbiome Sci Med. 2013;1(1):10-29.

82. Aagaard K, Riehle K, Ma J, Segata N, Mistretta T, Coarfa C, et al. A metagenomic approach to characterization of the vaginal microbiome signature in pregnancy. Plos One. 2012;7(6):1-15.

83. Romero R, Hassan S, Gajer P, Tarca A, Fadrosh D, Nikita L, et al. The composition and stability of the vaginal microbiota of normal pregnant women is different from that of non-pregnant women. Microbiome. 2014; 2(1):1-19.

84. Huang $Y$, Wang $Y$, He $Y$, Ji $Y$, Wang $L$, Sheng $H$, et al. Homogeneity of the vaginal microbiome at the cervix, posterior fornix, and vaginal canal in pregnant Chinese women. Microb Ecol. 2014;69(2):407-14.

85. Mirmonsef $\mathrm{P}$, Hotton Al, Gilbert D, Burgad D, Landay A, Weber Km et al. Free glycogen in vaginal fluids is associated with Lactobacillus colonization and low vaginal pH. Plos One. 2014;9(7):1-11.

86. Holzapfel W, Wood B, editors. Lactic acid bacteria: biodiversity and taxonomy. Somerset: Wiley; 2014.

87. Harwich M, Serrano M, Fettweis J, Alves J, Reimers M, Buck G, et al. Genomic sequence analysis and characterization of Sneathia amnii sp. nov. BMC Genomics. 2012;13 Suppl 8:1-15.

88. Krieg N, Ludwig W, Euzéby J, Whitman W. Phylum Xiv. Bacteroidetes Phyl. Nov. In: Krieg N, Staley J, Brown D, Hedlund B, Paster B, Ward N, et al., editors. Bergey's Manual Of Systematic Bacteriology: Volume Four The Bacteroidetes, Spirochaetes, Tenericutes (Mollicutes), Acidobacteria, Fibrobacteres, Fusobacteria, Dictyoglomi, Gemmatimonadetes, Lentisphaerae, Verrucomicrobia, Chlamydiae, And Planctomycetes. New York: Springer New York; 2010. p. 25-469.

89. Eschenbach D, Thwin S, Patton D, Hooton T, Stapleton A, Agnew K, et al. Influence of the normal menstrual cycle on vaginal tissue, discharge, and microflora. Clin Infect Dis. 2000;30(6):901-7.

90. Mirmonsef P, Gilbert D, Veazey R, Wang J, Kendrick S, Spear G. A comparison of lower genital tract glycogen and lactic acid levels in women and macaques: implications for HIV and SIV susceptibility. AIDS Res Hum Retrov. 2012;28(1):76-81.

91. Lockhart A, Thrall P, Antonovics J. Sexually transmitted diseases in animals: ecological and evolutionary implications. Biol Rev. 1996;71(3):415-71.

92. Smith G, Dobson A. Sexually-transmitted diseases in animals. Parasitol Today. 1992;8(5):159-66.

93. Klaenhammer T. Bacteriocins of lactic acid bacteria. Biochimie. 1988;70(3): 337-49.

94. Kalyoussef S, Nieves E, Dinerman E, Carpenter C, Shankar V, Oh J, et al. Lactobacillus proteins are associated with the bactericidal activity against $E$. coli of female genital tract secretions. Plos One. 2012;7(11):1-9.

95. Mc A, Artis D. The dynamic influence of commensal bacteria on the immune response to pathogens. Curr Opin Microbiol. 2013;16(1):4-9.

96. Fitzpatrick C, Altmann J, Alberts S. Exaggerated sexual swellings and male mate choice in primates: testing the reliable indicator hypothesis in the Amboseli baboons. Anim Behav. 2015;104:175-85.

97. Clarke P, Barrett L, Henzi S. What role do olfactory cues play in chacma baboon mating? Am J Primatol. 2009;71(6):493-502.

98. Archie $\mathrm{E}$, Theis K. Animal behaviour meets microbial ecology. Anim Behav. 2011;82:425-36.

99. Ezenwa V, Williams A. Microbes and animal olfactory communication: where do we go from here? Bioessays. 2014:36(9):847-54.

100. Doty R, Ford M, Preti G, Huggins G. Changes in the intensity and pleasantness of human vaginal odors during the menstrual cycle. Science. 1975;190:1316-8.

101. Funkhouser L, Bordenstein S. Mom knows best: the universality of maternal microbial transmission. Plos Biol. 2013;11(8):1-9.

102. Smith C, Mueller U. Sexual transmission of beneficial microbes. Trends Ecol Evol. 2015;30(8):438-40.

103. Thrall P, Antonovics J, Dobson A. Sexually transmitted diseases in polygynous mating systems: prevalence and impact on reproductive success. P Roy Soc Lond B Bio. 2000;267(1452):1555-63.

104. Kokko H, Ranta E, Ruxton G, Lundberg P. Sexually transmitted disease and the evolution of mating systems. Evolution. 2002;56(6):1091-100.

105. Lombardo $M$, Thorpe $P$, Power $H$. The beneficial sexually transmitted microbe hypothesis of avian copulation. Behav Ecol. 1999;10(3):333-7.

106. Smart S, Singal A, Mindel A. Social and sexual risk factors for bacterial vaginosis. Sex Transm Infect. 2004;80(1):58-62.

107. Schwebke J, Desmond R. Risk factors for bacterial vaginosis in women at high risk for sexually transmitted diseases. Sex Transm Dis. 2005;32(11):654-8.

\section{Submit your next manuscript to BioMed Central and we will help you at every step:}

- We accept pre-submission inquiries

- Our selector tool helps you to find the most relevant journal

- We provide round the clock customer support

- Convenient online submission

- Thorough peer review

- Inclusion in PubMed and all major indexing services

- Maximum visibility for your research

Submit your manuscript at www.biomedcentral.com/submit
) Biomed Central 